\title{
A Strontium Ranelate Incorporated Enzyme-crosslinked Gelatin Nanoparticle/Silk Fibroin Aerogel for Osteogenesis in OVX-induced Osteoporosis
}

\section{Yihan Li, Dize Li, Tao Chen, Yuanding Huang}

Stomatological Hospital of Chongqing Medical University

Chongqing Key Laboratory of Oral Diseases and Biomedical Sciences

\section{Objective}

Osteoporosis is a wide-range disease with negative impact on bone defects healing. Strontium ranelate (SR) has promising osteogenic potential for its dual function on stimulating osteoblasts and inhibiting osteoclasts activity. However, it has limitations for its dose-dependent effect and side effects on systemic application. Here, a sequentially crosslinking strategy including enzyme-crosslinking through tyrosinase from mushroom and physical folding is acquired to create SR loaded gelatin nanoparticle/silk fibroin aerogel (abbreviated as S/G-Sr-MT) with drug release controlling capacity.

\section{Experimental section}

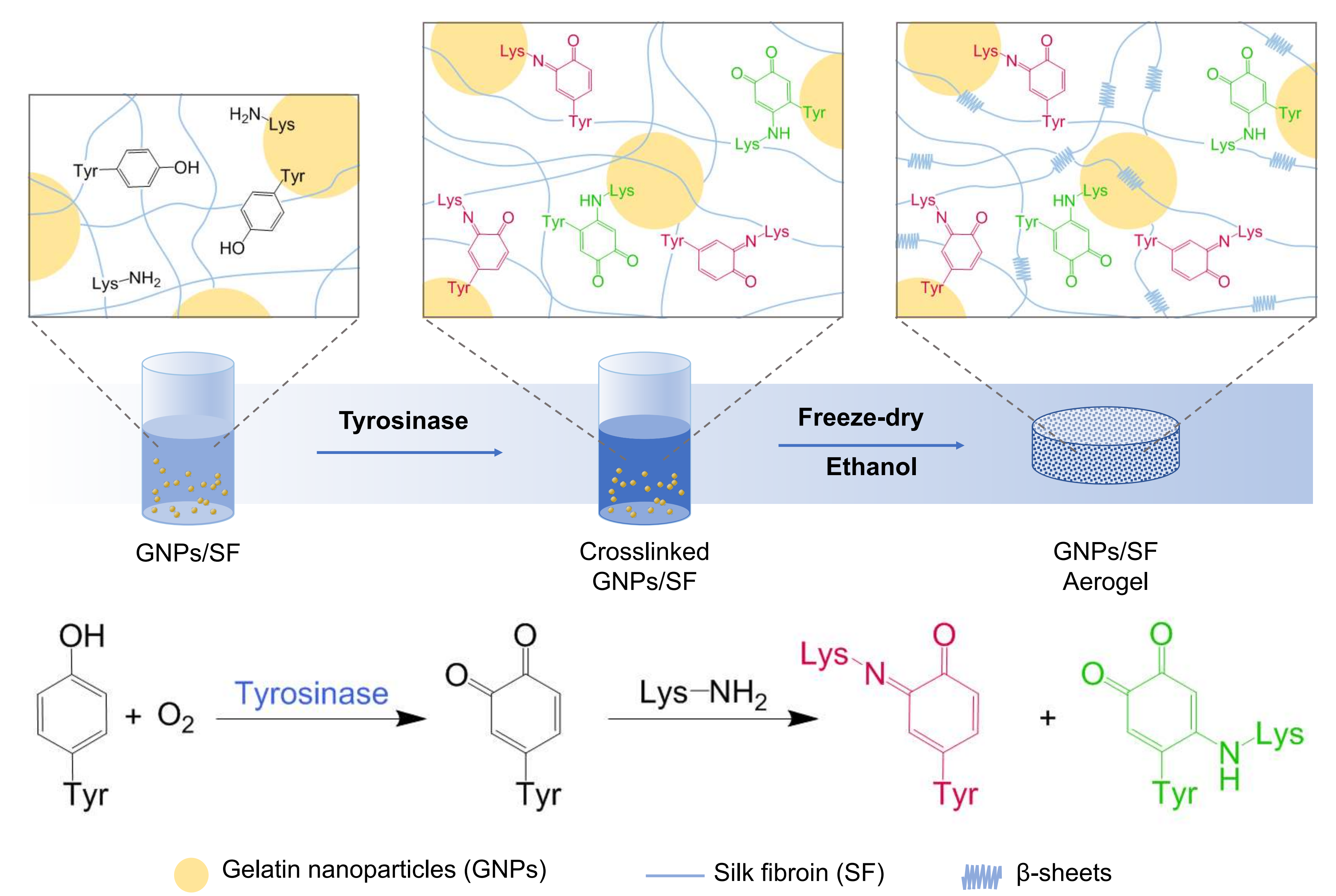

Based on a sequential enzyme-crosslinking strategy, we fabricated a composite aerogel scaffold assembled from SF and SR loaded GNPs (denoted as S/G-Sr-MT) for OVX bone regeneration. We used ninhydrin test and synchronous fluorescence analysis to examine the effect of sequentially-crosslinking. The morphology and properties of S/G-Sr-MT scaffold were evaluated and the release behavior of $\mathrm{Sr}^{2+}$ was explored. In vitro experiment, the mouse embryo osteoblast precursor cells linage (MC3T3-E1) were seeded on S/G-SrMT and the cell proliferation, morphology and osteogenic differentiation were detected. In vivo experiment, we implanted the composite scaffolds into calvaria defects of OVX rats to examine its bone healing capability.

\section{Results}
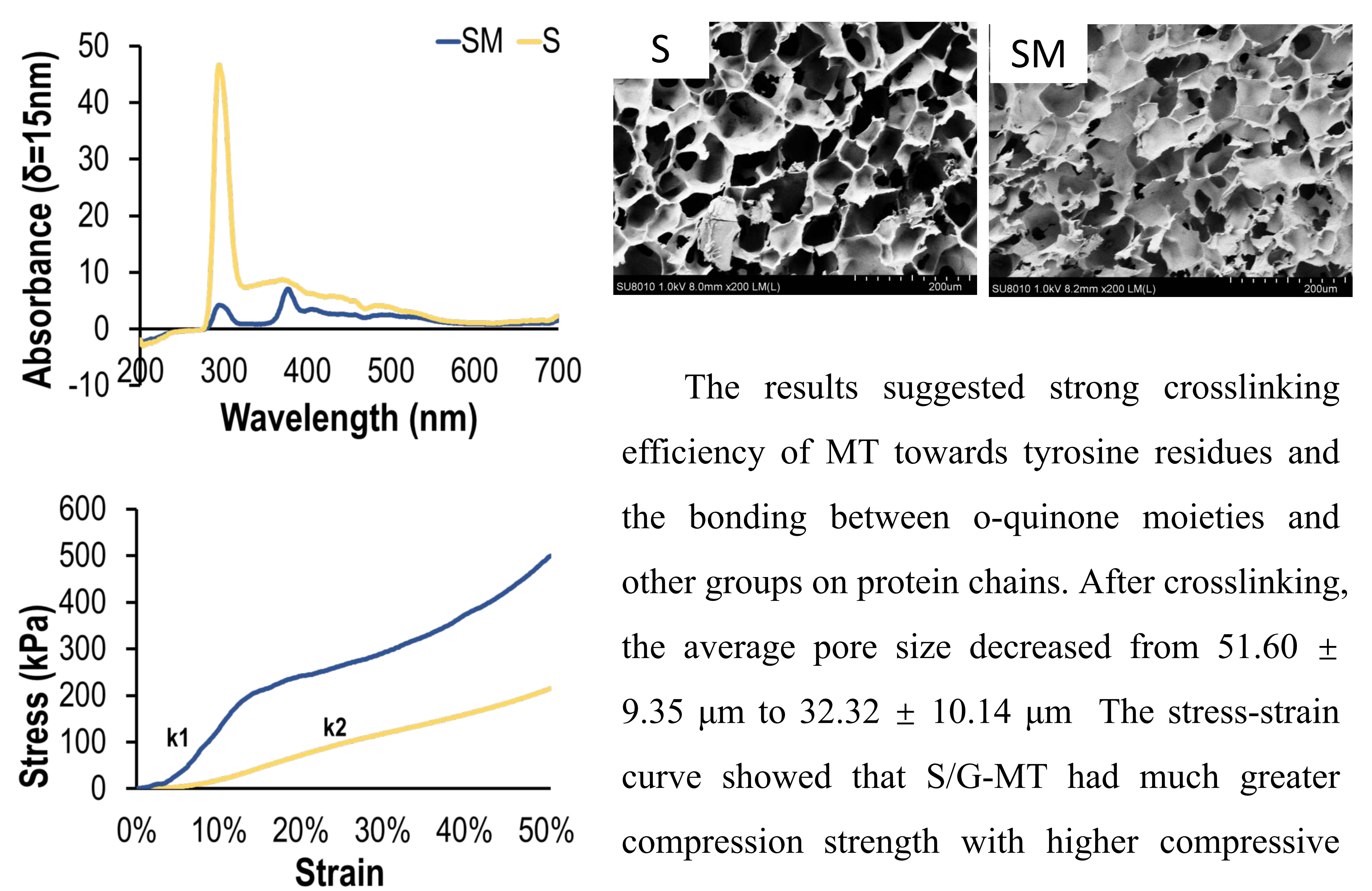

The results suggested strong crosslinking efficiency of MT towards tyrosine residues and the bonding between o-quinone moieties and other groups on protein chains. After crosslinking, the average pore size decreased from $51.60 \pm$ $9.35 \mu \mathrm{m}$ to $32.32 \pm 10.14 \mu \mathrm{m}$ The stress-strain curve showed that S/G-MT had much greater compression strength with higher compressive modulus.
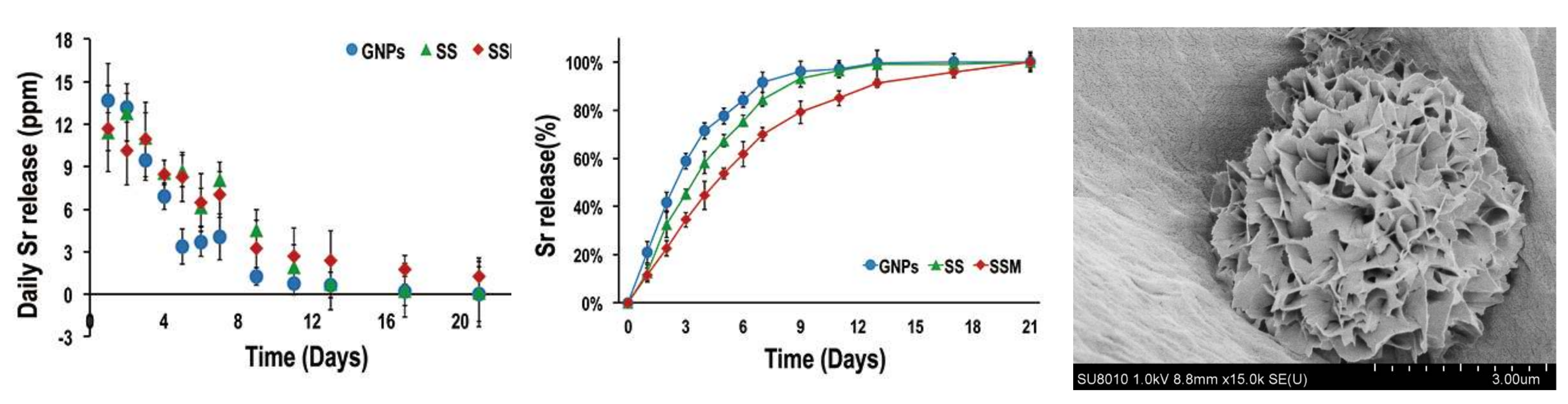
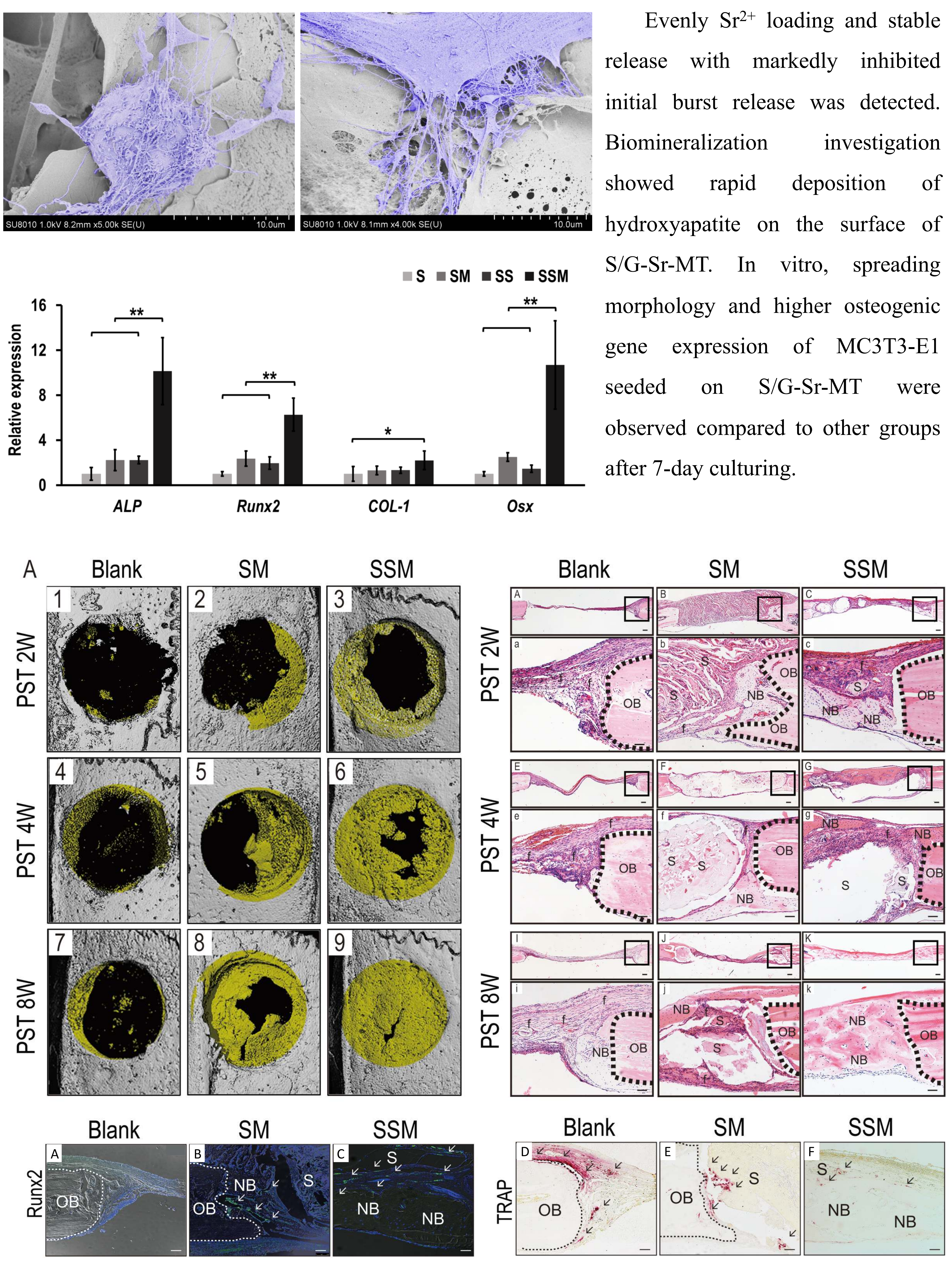

In vivo, S/G-Sr-MT showed obvious osteogenic capacity in calvaria defects of ovariectomized rats in which high Runx2 expression and inhibited TRAP activity were observed. Such results suggested the S/G-Sr-MT scaffold could stimulate osteogenic differentiation of osteoblasts while inhibit osteoclast behaviors in vivo. These findings highlight the potential osteogenic ability and clinical application of SR incorporated enzymecrosslinked scaffold in ovariectomized (OVX) bone healing.

\section{Conclusion}

In this assay, to promote new bone formation of OVX bone defects, the composite matrix of GNPs and SF was created through sequentially enzyme-crosslinking and physical folding. This effective and mild crosslinking method had modified the interior structure and improved mechanical properties of this aerogels. The preponderant bone formation boosting capacity proved that this aerogel is a promising bone tissue engineering material. 